\title{
CẢI CÁCH CHƯƠNG TRÌNH GIẢNG DẠY THEO MÔ HİNH CDIO NHẰM MANG LẠI VIỆC HỌC CHỦ ĐộNG CHO NGƯỜI HỌC BÀ̀NG PHƯƠNG PHÁP TẠO CộNG ĐỒNG DẠY HỌC
}

\author{
Lê Thanh Lành ${ }^{(*)}$, Nguyễn Hũu Huy ${ }^{(* *)}$ \\ (*) Tiến sĩ. Truoòng Đại học Công nghệ Đồng Nai. Email: lathanhlanh@dntu.edu.vn. \\ (**) Thạc sĩ. Truờng Đại học Công nghệ Đồng Nai. Email: nguyenhuuhuy@dntu.edu.vn
}

DOI: $\underline{10.37550 / \text { tdmu.CFR/2021.1.135 }}$

\section{Tóm tắt}

Bài viết này trình bày về cộng đồng giảng dạy học tập tích cực đurợc tạo ra để hỗ trợ việc thực hiện giai đoạn cải cách chuoong trình giảng dạy theo mô hình CDIO. Cộng dồng mở cửa cho tất cả các giảng viên kỹ thuật. Cộng đồng giảng dạy này khuyến khich việc sủ dụng học tập tích cực và công nghệ thông tin trong lớp học và cung cấp cho nguời huớng dẫn một khuôn khổ ngang hàng để hỗ trọ họ trong khi hình thành, thiết kế, thưc hiện và đánh giá nhũng đổi mới trong dạ học. Nó tuân theo một mô hình hỗ trợ việc chuyển giao các kinh nghiệm thành công qua các khóa học trong một chuơng trình và cả các chuơng trình kỹ thuật. Một trong nhũng thành tưu chính là giúp cải thiện giao tiếp và tăng cuờng hợp tác giữa các giảng viên, được hỗ trọ trong việc giám sát và đánh giá việc thực hiện cải cách chuoong trình giảng dạy, được truyền cảm hưng nghiên cưu về giáo dục kỹ thuật giữa các thành viên và đóng góp cho khoa kế hoạch phát triển mà họ đang được thục hiện.

Từ khóa: học tập tích cục, cộng đồng giảng dạy, cải cách chuơng trình giảng dạy theo mô hình CDIO, giáo dục kỹ thuật, nâng cao chất luợng giảng dạy của giảng viên

\section{1. Đặt vấn đề}

Bài viết này mô tả cộng đồng giảng dạy học tập tích cực được tạo ra để hỗ trợ giai đoạn thực hiện cải cách chương trình giảng dạy theo mô hình CDIO hiện đang được tiến hành tại Trường Đại học Công nghệ Đồng Nai[1]-[5]. Trường đại học của chúng tôi đã thiết lập mô hình tổ chức sư phạm, lấy con người làm trung tâm dựa trên bốn nền tảng: chương trình học dựa trên kết quả và năng lực, quá trình dạy và học lấy sinh viên làm trung tâm, giáo dục dựa trên đạo đức và đối thoại.

Giữa niềm tin và lý trí, và sự hòa nhập giữa học thuật và xã hội. Mô hình này được tóm tắt trong hình 1 . 


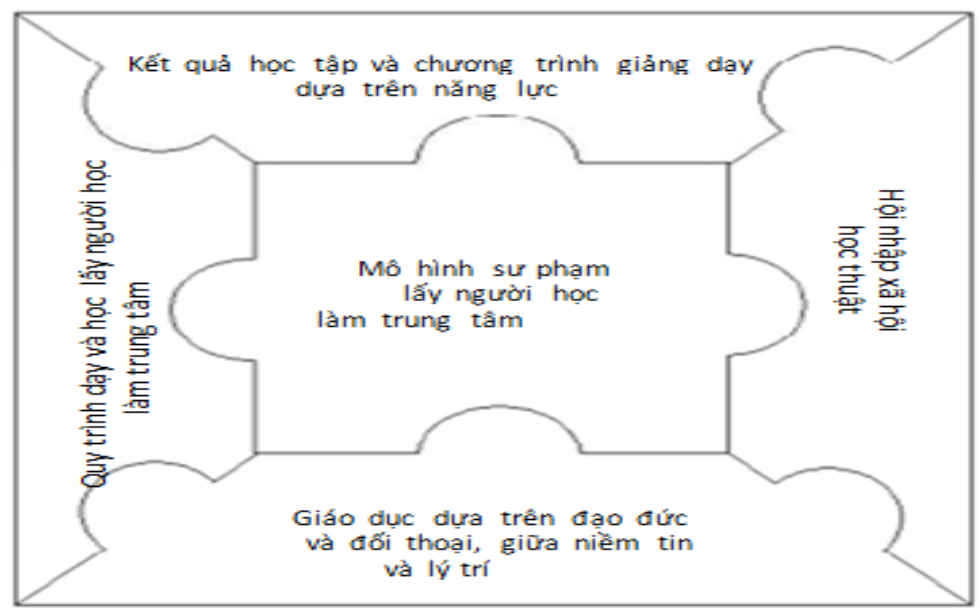

Hình 1. Mô hình tổ chức su phạm

Với mục đích này, trường đại học bắt buộc cải cách chương trình giảng dạy xuyên suốt tất cả các chương trình, và đào tạo giảng viên trong quá trình giảng dạy và học tập lấy sinh viên làm trung tâm. Hai quá trình này đã được thực hiện dần dần và tiến bộ. Để hỗ trợ các quy trình này, một trung tâm hỗ trợ phát triển các kỹ năng giảng dạy và thúc đẩy các đổi mới trong quy trình dạy và học lấy sinh viên làm trung tâm. Trung tâm này cung cấp một chương trình kỹ năng giảng dạy chứng nhận năng lực giảng viên toàn thời gian và bán thời gian trong năm. Mỗi năng lực được chứng nhận thông qua 40 giờ làm việc, bao gồm hội thảo, thực hiện mô hình trong một khóa học cụ thể, được theo dõi và hướng dẫn định kỳ trong suốt một học kỳ, và một báo cáo cuối cùng. Bảng 1 trình bày mô tả ngắn gọn về từng chứng nhận.

Bảng 1. Chưong trình kỹ năng giảng dạy

\begin{tabular}{|c|c|c|}
\hline Cấp độ & Chứng nhận & Miêu tả \\
\hline Cấp độ 1 & $\begin{array}{l}\text { Thiết kế khóa học dựa trên kết } \\
\text { quả học tập }\end{array}$ & $\begin{array}{l}\text { Đào tạo giảng viên thiết kế các khóa học dựa trên kết quả } \\
\text { học tập và phát triển các chương trình và giáo trình khóa } \\
\text { học sử dụng phương pháp lấy sinh viên làm trung tâm. }\end{array}$ \\
\hline Cấp độ 2 & Phương pháp học tập tích cực & $\begin{array}{l}\text { Đào tạo giảng viên về lý thuyết và thực hành một số } \\
\text { phương pháp học tập tích cực. }\end{array}$ \\
\hline Cấp độ 3 & Đánh giá kết quả học tập & Đào tạo giảng viên về các kỹ thuật đánh giá kết quả học tập. \\
\hline Cấp độ 4 & $\begin{array}{l}\text { Sử dụng công nghệ thông tin } \\
\text { trong quá trình dạy và học }\end{array}$ & $\begin{array}{l}\text { Hướng dẫn giảng viên cách phát triển các chiến lược giảng } \\
\text { dạy và ứng dụng hiệu quả công nghệ thông tin vào quá trình } \\
\text { học tập. }\end{array}$ \\
\hline Cấp độ 5 & Giảng dạy cộng đồng & $\begin{array}{l}\text { Thúc đẩy việc trao đổi kinh nghiệm giảng dạy giữa các } \\
\text { giảng viên vầ hệng thóng các đổi mới giảng dạy của họ. }\end{array}$ \\
\hline
\end{tabular}

Tại Trường Kỹ thuật, quá trình cải cách chương trình giảng dạy tuân theo cách tiếp cận dựa trên CDIO, có tính đến mô hình sư phạm và các tiêu chí công nhận kỹ thuật quốc gia. Phương pháp này được áp dụng, các giai đoạn đã được hoàn thành cho đến nay. Bài viết này cho thấy sự gắn kết sâu rộng giữa mô hình sư phạm và các tiêu chuẩn CDIO. 


\subsection{Tăng cuờng giảng viên}

Trong suốt quá trình thiết kế chương trình giảng dạy, Trường Kỹ thuật đã thúc đẩy sự phát triển và cấp chứng chỉ cho giảng viên, đặc biệt là trong hai năng lực sư phạm đầu tiên, như được mô tả và được minh họa trong Hình 2 .

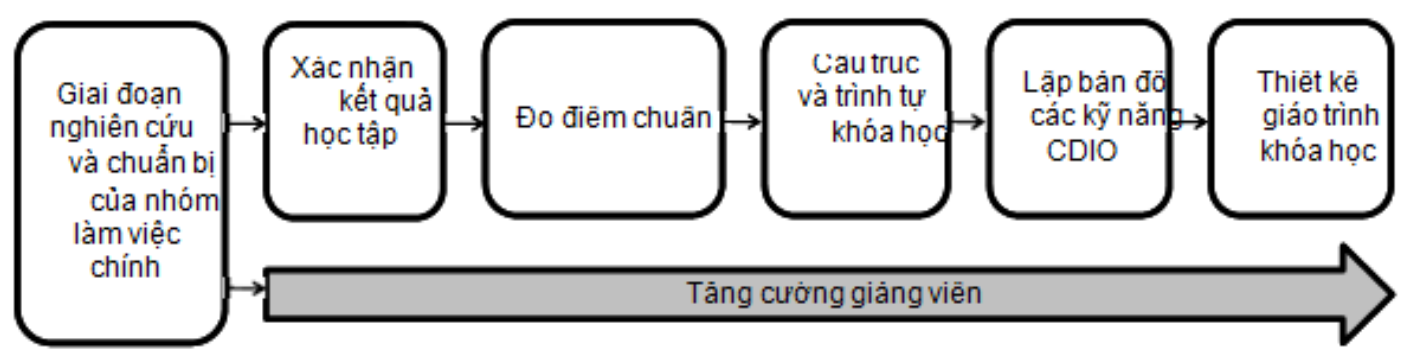

Hình 2. Nâng cao đội ngũ giảng viên trong suốt quá trình thiết kế chương trình giảng dạy

Ban đầu, việc nâng cao đội ngũ giảng viên này tập trung vào đội ngũ thiết kế chương trình giảng dạy và giảng viên toàn thời gian và bán thời gian năm thứ nhất. Quá trình này đã được mở rộng dần dần.

\subsection{Học tập tích cục}

Là một phần của quá trình cải cách chương trình giảng dạy tại Trường Kỹ thuật, học tập tích cực (tiêu chuẩn CDIO 8) đã được đưa vào chương trình giảng dạy. Một số kỹ thuật học tập tích cực $[6,7,8]$ đã được sử dụng, chẳng hạn như học tập dựa trên vấn đề và dự án, nghiên cứu điển hình, thảo luận nhóm nhỏ, câu hỏi khái niệm, tranh luận, thuyết trình, ghi nhớ phản chiếu, động não, lập bản đồ khái niệm, minilabs, vấn đề bộ, trong số những bộ khác.

\section{Mô hình giảng dạy cộng đồng}

Một cộng đồng giảng dạy như một hiệp hội tự nguyện của các giảng viên có chung sở thích làm việc cùng nhau để thúc đẩy việc cải tiến liên tục các phương pháp thực hành sư phạm thông qua việc trao đổi kinh nghiệm giảng dạy và học tập giữa họ và với các cộng đồng giảng dạy khác

Cộng đồng giảng dạy đầu tiên tại Trường Kỹ thuật được chính thức thành lập bao gồm các thành viên của khoa Khoa học Máy tính và Kỹ thuật Công nghiệp. Sau đó có thêm các thành viên của bộ phận Kỹ thuật Xây dựng. Cộng đồng giảng dạy được thiết kế để cung cấp cho giảng viên một khung đồng đẳng để hỗ trợ họ trong việc hình thành, thiết kế, thực hiện và đánh giá những đổi mới trong giảng dạy. Mục tiêu của nó là:

1. Thúc đẩy việc học tập tích cực trong các chương trình giảng dạy kỹ thuật và để hỗ trợ họ trong khi hình thành, thiết kế, thực hiện và đánh giá các đổi mới trong giảng dạy.

2. Tận dụng cộng đồng đồng đẳng để thúc đẩy sự phát triển của giảng viên trong các phương pháp học tập tích cực. 
3. Hỗ trợ việc chuyển giao kinh nghiệm thành công qua các chuỗi khóa học trong một chương trình và cả các chương trình kỹ thuật.

4. Thúc đẩy việc sử dụng công nghệ thông tin trong lớp học.

Mô hình Cộng đồng Giảng dạy được thể hiện trong Hình 3. Trong mô hình này, giáo viên thiết kế, áp dụng và đánh giá các hoạt động ngoại khóa cho mỗi khóa học bằng cách sử dụng học tập tích cực và các nguồn CNTT sẵn có (mục tiêu 4). Cộng đồng giảng dạy khuyến khích giáo viên ghi lại trải nghiệm và tạo ra bằng chứng về kết quả của họ, và chia sẻ chúng với các đồng nghiệp của họ để nhận được phản hồi về hoạt động ngoại khóa và do đó cải thiện và hệ thống hóa các đổi mới sư phạm của họ (mục tiêu 1). Trọng tâm của mô hình cộng đồng giảng dạy nằm ở các cuộc họp, nơi các thành viên củng cố quy trình cải tiến liên tục này bằng cách đưa ra phản hồi và giúp các thành viên cải thiện các đổi mới trong giảng dạy và bằng cách giám sát việc tuân thủ các mục tiêu chương trình của Trường Kỹ thuật (mục tiêu 1 và 2).

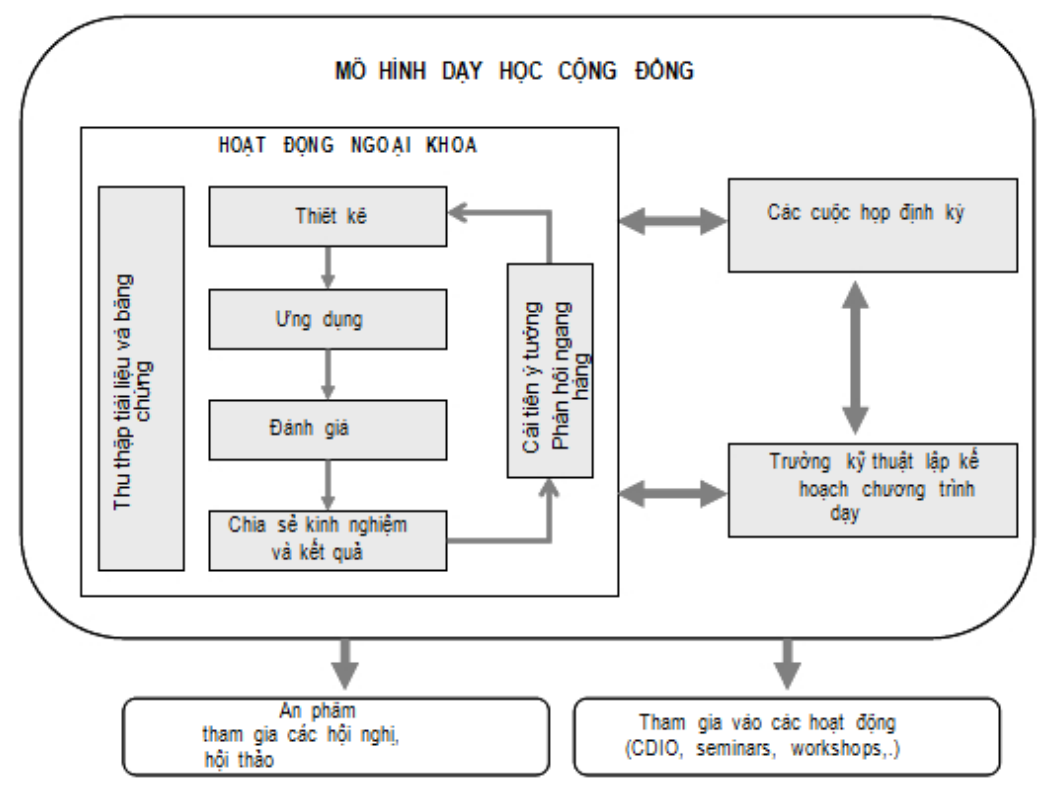

Hinh 3. Mô hìn giảng dạy công đồng

Ngoài ra, cộng đồng giảng dạy cung cấp những đổi mới giảng dạy thông qua việc tham gia tích cực vào các hoạt động cấp trường như hội thao giảng dạy và hội thảo. Đồng thời, cộng đồng còn thúc đẩy việc công bố kết quả trong các hội nghị, hội thảo và tạp chí trong giáo dục kỹ thuật (mục tiêu 3).

\section{Các hoạt động và kết quả dạy học cộng đồng}

Để đạt được mục tiêu, kế hoạch thiết kế ban đầu cho cộng đồng giảng dạy đã chỉ rõ một số hoạt động: Bao gồm thúc đẩy học tập tích cực trong các khóa học kỹ thuật từ năm đầu tiên trở đi, và chuyển giao kinh nghiệm thành công qua các khóa học và các chương trình kỹ thuật, do đó thúc đẩy các giảng viên khác áp dụng phương pháp học tập tích cực trong các khóa học của họ. Các hoạt động này được mô tả chi tiết trong các đoạn sau. 


\section{Hoạt động 1: Học tập tích cục trong các khóa học kỹ sư năm thứ nhất}

Như đã đề cập trước đây, học tập tích cực (tiêu chuẩn CDIO 8) đã được đưa vào chương trình giảng dạy kỹ thuật. Đặc biệt, khối lượng khóa học năm đầu tiên của chương trình khoa học máy tính bao gồm hai khóa học nhập môn kéo dài một học kỳ, như thể hiện trong Hình 4.

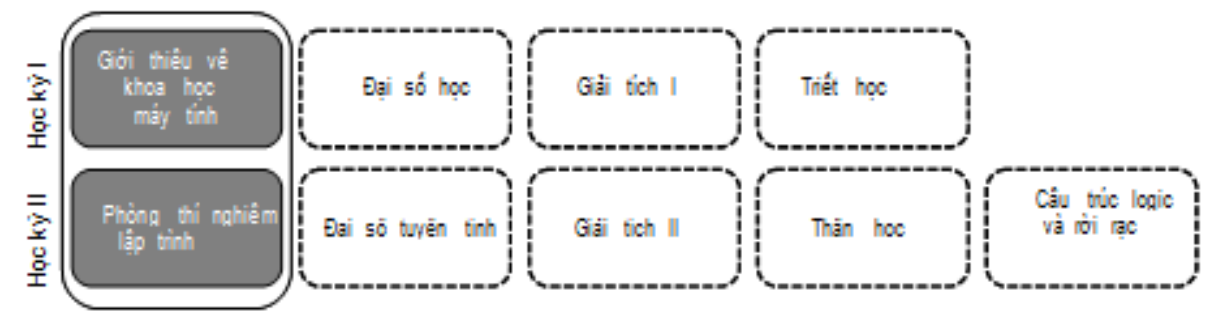

Hinh 4. Khóa học khoa học máy tính năm đầu tiên

Trong khóa học Nhập môn Khoa học Máy tính, sinh viên làm quen với lĩnh vực và vai trò nghề nghiệp đã chọn của họ và vòng đời của phần mềm bằng cách phát triển một dự án từ khi hình thành đến hoạt động của nó. Khóa học này phát triển các kỹ năng như kỹ năng giao tiếp, lập kế hoạch, xây dựng mô hình, xây dựng chiến lược giải quyết vấn đề, phân tích phản biện và làm việc nhóm. Khóa học thứ hai là Phòng thí nghiệm lập trình, nơi các nhóm sinh viên phân tích các vấn đề khoa học máy tính và thiết kế các giải pháp theo cách tiếp cận có cấu trúc. Khóa học này cho phép sinh viên tham gia vào lập trình và để phát triển các kỹ năng cá nhân để tự học và làm việc nhóm.

Khối lượng khóa học năm đầu tiên của chương trình kỹ thuật công nghiệp bao gồm hai khóa học giới thiệu thời lượng học kỳ, như thể hiện trong Hình 5.

Khóa học Nhập môn Kỹ thuật Công nghiệp chuẩn bị cho sinh viên vào cuộc sống học tập của họ bằng cách cung cấp cho họ các công cụ cần thiết để hiểu tầm nhìn, hoạt động và kỹ năng giải quyết vấn đề của một kỹ sư công nghiệp, xem xét nền tảng khoa học và cơ sở công nghệ trong lĩnh vực hoạt động của họ, đồng thời trau dồi khả năng phân tích vấn đề và đề xuất giải pháp thông qua các quá trình ra quyết định có hệ thống. Nó cũng phát triển các kỹ năng lập kế hoạch làm việc độc lập và làm việc nhóm, đồng thời cung cấp cho sinh viên các công cụ cơ bản để cải thiện kỹ năng giao tiếp của họ.

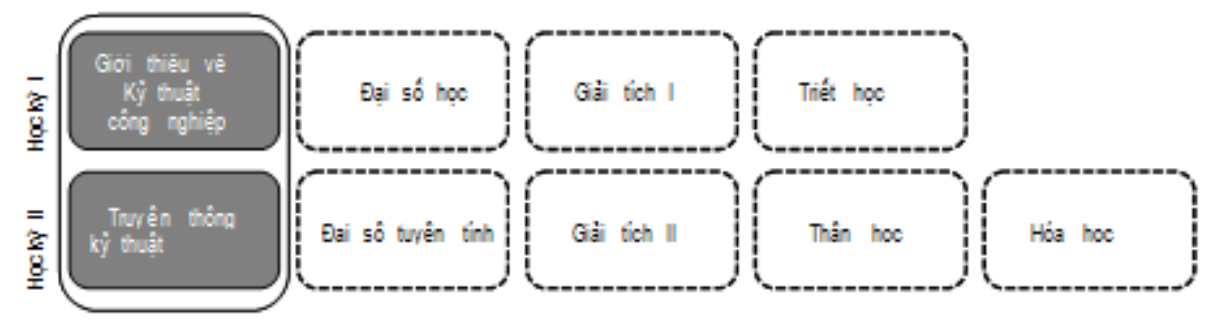

Hinh 5. Tải trọng khóa học năm đầu tiên ngành kỹ thuật công nghiệp

Khóa học Kỹ thuật Giao tiếp trong học kỳ thứ hai cung cấp cho sinh viên một số kỹ năng giao tiếp, đặc biệt là kỹ năng diễn đạt và sử dụng các công cụ hiển thị đồ họa. Sinh viên cũng được đào tạo về các công cụ cơ bản để lập kế hoạch dự án. 
Hoạt động này đóng góp vào các mục tiêu $1,2,3$ và 4 của cộng đồng giảng dạy và kết quả chính của nó là:

• "Giới thiệu về Kỹ thuật trong bối cảnh Cải cách Ngoại khóa CDIO”.

- “Học tập tích cực trong các khóa học kỹ thuật năm đầu tiên” .

- Sinh viên đánh giá cao việc sử dụng công nghệ thông tin. Bảng 2 cho thấy tỷ lệ sinh viên đánh giá tích cực việc sử dụng các công cụ CNTT trong các khóa học năm thứ nhất để đạt được kết quả học tập. Việc sử dụng các công cụ CNTT trong kỹ thuật công nghiệp năm đầu tiên các khóa học đã được khảo sát vào cuối năm. Khóa học Phòng thí nghiệm lập trình được cung cấp hai lần mỗi năm.

- Thiết kế một hội thảo học tập tích cực cho cuộc họp CDIO. Hội thảo này sẽ trình bày việc áp dụng phương pháp Phân tích - Thiết kế - Lập trình - Kiểm tra (ADPT) trong Phòng thí nghiệm Lập trình khóa học.

Bảng 2. Tỷ lệ sinh viên đánh giá tích cực việc sủ dụng các công cụ CNTT, mỗi khóa học

\begin{tabular}{|c|l|c|c|}
\hline \multicolumn{2}{|c|}{ Các khóa học và các công cụ công nghệ thông tin của họ } & 2018 & 2019 \\
\hline \multirow{2}{*}{ Khoa học máy tính } & Giới thiệu về khoa học máy tính & $56 \%$ & $83 \%$ \\
\cline { 2 - 4 } & Phòng thí nghiệm lập trình & $52 \%$ & $82 \%$ \\
\hline \multirow{2}{*}{$\begin{array}{c}\text { Kỹ thuật công } \\
\text { nghiệp }\end{array}$} & Giới thiệu về kỹ thuật công nghiệp & $81 \%$ & $83 \%$ \\
\cline { 2 - 4 } & Truyền thông kỹ thuật & $81 \%$ & $80 \%$ \\
\hline
\end{tabular}

\section{Hoạt động 2: Chuyển giao kinh nghiệm thành công qua các chuỗi khóa học}

\section{Khoa học máy tính}

Hai lần một học kỳ, giảng viên chịu trách nhiệm về các khóa học trong trình tự Lập trình của các khóa học được trình bày trong Hình 6 gặp nhau để chia sẻ kinh nghiệm và thảo luận về các chiến lược nhằm giải quyết các vấn đề chung.

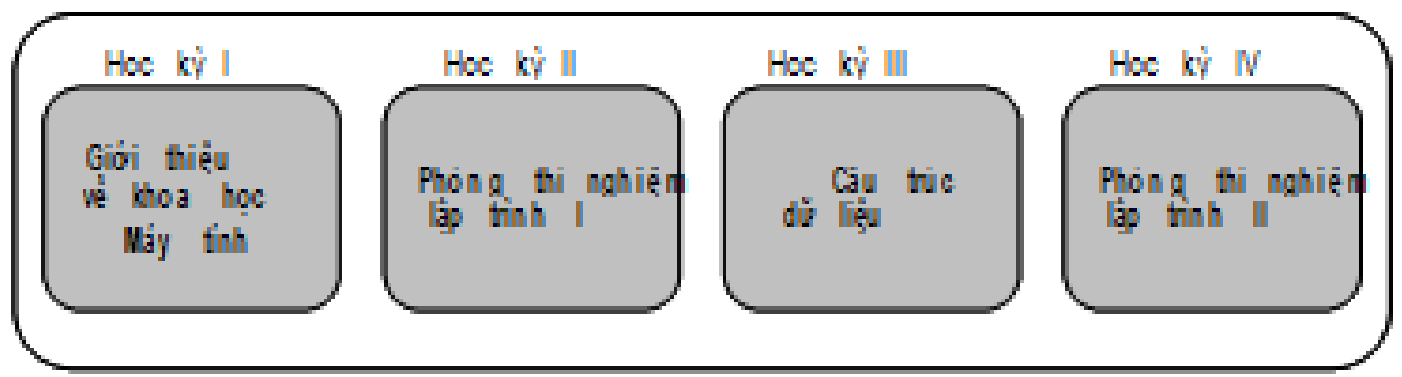

Hinh 6. Trình tụ khoa học máy tính của các khóa học lập trình (Hình bị nhòe)

\section{Kỹ thuật công nghiệp}

Các giảng viên chịu trách nhiệm về Giới thiệu về Kỹ thuật Công nghiệp và các khóa học Truyền thông Kỹ thuật được trình bày trong Hình 5 bao gồm các thành viên của Khoa 
Kỹ thuật Công nghiệp, họp định kỳ để thảo luận về sự tiến bộ của sinh viên và phối hợp các hoạt động để giúp sinh viên đạt được kết quả học tập như mong đợi.

\section{Công trình dân dụng}

Trong vài năm qua, các giảng viên chịu trách nhiệm về phần đầu tiên của trình tự kỹ thuật kết cấu của các khóa học của chương trình Kỹ thuật xây dựng, đã họp không chính thức để thảo luận về tiến bộ của sinh viên và chia sẻ kinh nghiệm và đổi mới sư phạm của họ. Theo kết quả đầu tiên của các cuộc họp này, trình tự kỹ thuật kết cấu đã được xác định lại theo cách phi thông thường, như thể hiện trong Hình 7 , trong bối cảnh cải cách chương trình giảng dạy của Trường Kỹ thuật.

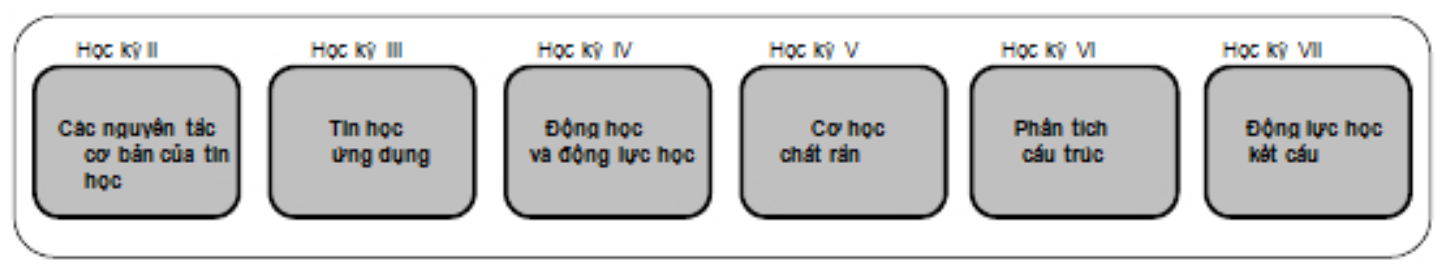

Hình 7. Trình tụ kỹ thuật xây dụng của các khóa học kỹ thuật kết cấu

Được thúc đẩy bởi kinh nghiệm của cộng đồng giảng dạy này, các giảng viên đang lên kế hoạch tạo cộng đồng giảng dạy của riêng họ tập trung vào trình tự kỹ thuật kết cấu hoàn chỉnh của các khóa học.

Mục tiêu đầu tiên sẽ là chính thức hóa và hệ thống hóa công việc của nhóm này, về việc theo dõi sự tiến bộ của sinh viên và kết quả học tập mong đợi của sinh viên trong toàn bộ chuỗi khóa học. Mục tiêu thứ hai là triển khai học tập tích cực trong tất cả các khóa học này, lấy đó làm mô hình học tập tích cực đã được sử dụng trong khóa học Cơ học truyền thống, cũng như các kinh nghiệm thành công khác.

\section{thuật \\ Hoạt động 3: Chuyển giao kinh nghiệm thành công qua các chương trình kỹ}

Kinh nghiệm của sinh viên từ khóa học Nhập môn Kỹ thuật Công nghiệp đã được chuyển thành công sang khóa học khác. Trong khóa học Giới thiệu về Kỹ thuật Công nghiệp, sinh viên tìm hiểu về lĩnh vực làm việc và vai trò của kỹ sư công nghiệp trong xã hội bằng cách tạo video theo nhóm, trong đó họ phỏng vấn các chuyên gia thực hành. Trong khóa học, sinh viên đã phỏng vấn một số chuyên gia cấp quản lý từ các doanh nghiệp liên quan để đối chiếu các yếu tố quản lý khái niệm với kinh nghiệm thực tế và các bài thuyết trình video chuẩn bị đã được đánh giá sử dụng cùng một phiếu đánh giá được sử dụng trong khóa học Kỹ thuật Công nghiệp.

\section{Hoạt động 4: Điều phối các hội thảo chuyên sâu}

Tốc độ tham gia của các giảng viên Trường Kỹ thuật vào khóa học cung cấp trong Bảng 1 chậm đi đáng kể. Vì điều này và được thúc đẩy bởi một hội thảo chuyên sâu do khoa Kỹ thuật Xây dựng tổ chức, cộng đồng giảng dạy đã phối hợp với một hội thảo chuyên sâu hai ngày về giảng dạy các khóa học của chương trình kỹ năng tập trung vào năng lực cấp độ 2 đến cấp độ 4 , cho Khoa học Máy tính và khoa Kỹ thuật Công nghiệp, bao gồm các giảng viên của chương trình $K y ̃$ thuật Công nghiệp và Kỹ thuật khác. Kết quả là, 
sự tham gia khóa học CDIO đã tăng lên đáng kể, đạt khoảng $40 \%$ giảng viên Kỹ thuật của Trường. Đặc biệt, Bảng 3 cho thấy tỷ lệ giảng viên Khoa học Máy tính, Kỹ thuật Công nghiệp và $K y ̃$ thuật Xây dựng có đã được tham dự các hội thảo về CDIO trong các năng lực khác nhau của mức độ 1 đến mức độ 5 . Cần lưu ý rằng các giảng viên đã hoàn thành quá trình phát triển của họ sẽ chỉ được cấp chứng chỉ sau khi năng lực tương ứng được thực hiện trong các khóa học cụ thể sẽ hoàn thành.

Bảng 3. Tỷ lẹ giảng viên tham dụ hội thảo CDIO, theo khoa

\begin{tabular}{|c|c|c|c|}
\hline Mức độ & Khoa học máy tính & Kõ thuật công nghiệp & Công trình dân dụng \\
\hline 1 & $50 \%$ & $15 \%$ & $77 \%$ \\
\hline 2 & $50 \%$ & $62 \%$ & $77 \%$ \\
\hline 3 & $50 \%$ & $62 \%$ & $77 \%$ \\
\hline 4 & $33 \%$ & $69 \%$ & $77 \%$ \\
\hline 5 & $17 \%$ & $8 \%$ & $8 \%$ \\
\hline
\end{tabular}

\section{Thảo luận và công việc tương lai}

Động lực của mô hình cộng đồng giảng dạy là các cuộc họp định kỳ, phù hợp với kế hoạch hàng năm của cộng đồng. Chương trình của cuộc họp giải quyết các nhiệm vụ cụ thể để đạt được mục tiêu của cộng đồng và kết hợp đánh giá thường xuyên về kết quả và phản hồi của người học. Các cuộc họp này thường mang tính hỗ trợ, ngắn và hiệu quả. Điều này có thể là do các thành viên trong cộng đồng luôn năng động, cởi mở với những đổi mới trong giảng dạy và đã làm việc cùng nhau kể từ khi họ tham gia vào quá trình cải cách giáo trình cho các chương trình tương ứng của họ, vì vậy họ đã xây dựng lòng tin giữa họ. Họ cũng đã chia sẻ quá trình nâng cao giảng viên cùng nhau. Như vậy, cộng đồng giảng dạy tuân theo một tổ chức thứ bậc phẳng.

Mặt khác, cộng đồng giảng dạy vẫn còn hẹp và các thành viên chủ yếu là tự chọn. Trong nhiều trường hợp, họ là những nhà lãnh đạo được Trường trao quyền để lãnh đạo quá trình cải cách chương trình giảng dạy. Tuy nhiên, chưa có lãnh đạo nào tham gia vào cộng đồng. Mặc dù cộng đồng giảng dạy tích cực lập tài liệu và công bố các hoạt động và kết quả của mình, nhưng cộng đồng này vẫn chưa có chiến lược tiếp cận để thêm các thành viên mới, những người thờ ơ hoặc thậm chí phản đối sự thay đổi. Các kế hoạch trong tương lai cho cộng đồng giảng dạy bao gồm: Tăng số lượng thành viên để tạo điều kiện chuyển giao kinh nghiệm và đổi mới sư phạm bằng cách sử dụng học tập tích cực trong các khóa học và chương trình; truyền cảm hứng cho nghiên cứu về giáo dục kỹ thuật giữa các thành viên và thúc đẩy việc phổ biến các phát hiện của họ; và thiết lập mối quan hệ với các cộng đồng giảng dạy khác để thúc đẩy công việc liên ngành giữa các sinh viên. Ví dụ, các thành viên trong cộng đồng có thể tổ chức các cuộc hội thảo hai lần một năm và duy trì một trang web để hiển thị những đổi mới về sư phạm và có cơ hội nhận được phản hồi từ nhiều đối tượng hơn. Đồng thời, Trường Kỹ thuật cam kết bắt đầu kết hợp học tập dịch vụ như một phương pháp học tập và đã bắt đầu phát triển đội ngũ giảng viên cho mục đích này. Một số thành viên cộng đồng giảng dạy sẵn sàng làm việc trên các chiến lược để kết hợp hai cách tiếp cận này.

\section{Kết luận}


Mặc dù cộng đồng giảng dạy của chúng tôi còn tương đối mới và nhỏ, nhưng nó đã tạo ra tác động trong một số chương trình kỹ thuật bằng cách sử dụng học tập tích cực và kết hợp các công cụ công nghệ thông tin. Các thành viên của nó rất có động lực để cải thiện giao tiếp và tăng cường hợp tác giữa các giảng viên của các lĩnh vực khác nhau. Cộng đồng giảng dạy đã hỗ trợ trong việc giám sát và đánh giá việc thực hiện cải cách chương trình, và cũng đã đóng góp vào kế hoạch phát triển giảng viên. Mô hình dựa trên mối quan hệ đồng đẳng của cộng đồng nhằm thúc đẩy các phương pháp học tập tích cực đã truyền cảm hứng cho nghiên cứu về giáo dục kỹ thuật giữa các thành viên, mô hình này đã tạo ra một số ấn phẩm trong giảng dạy. Sự tham gia của cộng đồng vào mạng $\mathrm{CDIO}$ chắc chắn là một nguồn kiến thức và hướng dẫn vô giá.

\section{Tài liệu tham khảo}

[1] Nguyễn Thành Hải, Phùng Thúy Phượng, Đồng Thị Bích Thủy, 2010. Giới thiệu một số phương pháp giảng dạy cải tiến giúp sinh viên học tập chủ động và trải nghiệm, đạt các chuẩn đầu ra theo CDIO, Hội thảo CDIO 2010_Đại học Quốc Gia Tp.HCM.

[2] Hồ Tấn Nhựt, Đoàn Thị Minh Trinh (Biên dịch), 2009, Cải cách và xây dựng chương trình đào tạo kỹ thuật theo phương pháp tiếp cận CDIO, Nxb Đại học Quốc gia TP.HCM.

[3] Crawley, E., et al., "Rethinking Engineering Education: The CDIO Approach". Springer Sciences + Business Media LLC. New York, 2007.

[4] Loyer S., et al., "A CDIO approach to curriculum design of five engineering programs at UCSC", Proceedings of the 7th International CDIO Conference, Technical University of Denmark, Copenhagen, June 20-23, 2011.

[5] Muñoz M., et al., "Active Learning in first-year engineering courses at Universidad Católica de la Santísima Concepción, Chile", Proceedings of the 8th International CDIO Conference, Queensland University of Technology, Brisbane, July 1-4, 2012. 